Please do not remove this page

RMIT

UNIVERSITY

\title{
Novel flight management system for improved safety and sustainability in the CNS+A context
}

Ramasamy, Subramanian; Sabatini, Roberto; Gardi, Alessandro

https://researchrepository.rmit.edu.au/esploro/outputs/9921862622101341/filesAndLinks?institution=61RMIT_INST\&index=null

Ramasamy, S., Sabatini, R., \& Gardi, A. (2015). Novel flight management system for improved safety and sustainability in the CNS+A context. Proceedings of the 15th Integrated Communication, Navigation, and Surveillance Conference (ICNS), 2015, G3-1-G3-11. https://doi.org/10.1109/ICNSURV.2015.7121225 Document Version: Accepted Manuscript

Published Version: https://doi.org/10.1109/ICNSURV.2015.7121225

Repository homepage: https://researchrepository.rmit.edu.au (C) 2015 IEEE

Downloaded On 2023/04/26 21:30:13 +1000 
Thank you for downloading this document from the RMIT Research Repository.

The RMIT Research Repository is an open access database showcasing the research outputs of RMIT University researchers.

RMIT Research Repository:http://researchbank.rmit.edu.au/

\section{Citation:}

Ramasamy, S, Sabatini, R and Gardi, A 2015, 'Novel flight management system for improved safety and sustainability in the CNS+A context', in Proceedings of the 15th Integrated Communication, Navigation, and Surveillance Conference (ICNS), 2015, United States, 21-23 April 2015, pp. G3-1-G3-11.

See this record in the RMIT Research Repository at:

https://researchbank.rmit.edu.au/view/rmit:32004

Version: Accepted Manuscript

Copyright Statement: (C) 2015 IEEE

Link to Published Version:

http://dx.doi.org/10.1109/ICNSURV.2015.7121225 


\title{
NOVEL FLIGHT MANAGEMENT SYSTEM FOR IMPROVED SAFETY AND SUSTAINABILITY IN THE CNS+A CONTEXT
}

\author{
Subramanian Ramasamy, Roberto Sabatini and Alessandro Gardi \\ School of Aerospace, Mechanical and Manufacturing \\ RMIT University, Melbourne, VIC 3000, Australia \\ roberto.sabatini@rmit.edu.au
}

\begin{abstract}
Avionic system developers are faced with the challenge of researching and introducing innovative technologies that satisfy the requirements arising from the rapid expansion of global air transport while addressing the growing concerns for environmental sustainability of the aviation sector. As a consequence, novel systems are being developed in the Communication, Navigation and Surveillance/Air Traffic Management (CNS/ATM) and Avionics (CNS+A) context. The introduction of dedicated software modules in Next Generation Flight Management Systems (NG-FMS), which are the primary providers of automated navigation and guidance services in manned aircraft and Remotely-Piloted Aircraft Systems (RPAS), has the potential to enable the significant advances brought in by time based operations. In this paper, key elements of the NG-FMS architecture are presented that allow the incorporation of 4-Dimensional Trajectory (4DT) planning and optimisation with inclusion of CNS integrity monitoring and augmentation functions in the overall design. The NG-FMS is designed to be fully interoperable with a future ground based 4DT Planning, Negotiation and Validation (4-PNV) system, enabling automated Trajectory/Intent-Based Operations (TBO/IBO). The mathematical models for 4DT planning are presented and the CNS integrity performance criteria are identified for various mission- and safety-critical tasks. Evaluation of the proposed concepts and methodologies is performed through dedicated simulation test case. The results demonstrate the functional capability of the NG-FMS to generate cost-effective trajectory profiles satisfying operational as well as environmental constraints.
\end{abstract}

\section{Introduction}

Innovative Communication, Navigation and Surveillance/Air Traffic Management (CNS/ATM) technologies [1] are required to be developed and implemented in a phased manner to satisfy time based operations requirements. A fundamental means of attaining enhanced CNS performance and 4-Dimensional Trajectory (4DT) capabilities is the adoption of a Next Generation Flight Management System (NGFMS). Traditionally, the FMS has been an integral part of the avionics suite of airliners and military transport aircraft. In the future CNS and Avionics $(\mathrm{CNS}+\mathrm{A})$ context, the improvement in performance is enabled by the wide-spread availability of a number of diverse airborne sensors, systems and multi sensor data fusion techniques. From a strategic point of view, the introduction of a new ATM regulatory framework and new systems for Trajectory/Intent-Based Operations (TBO/IBO) requires substantial Research and Development (R\&D) activities. They include the development of innovative methods/algorithms for dynamic allocation of airspace resources and the development of NG-FMS for Remotely Piloted Aircraft Systems (RPAS) enabling the unrestricted access of these aerial robots to all classes or airspace [2]. In the CNS+A context, the first recommendation addressing the operational and certification issues for civil UAS was issued by the Joint Aviation Authorities (JAA) CNS/ATM Steering Group $[3,4]$. Subsequently, the EUROCAE Working Group (WG-73) endeavours to address the following aspects:

a. RPAS operations enabled by cooperative and non-cooperative Sense-and-Avoid (SAA) solutions.

b. Command, control, communication, spectrum and security issues.

c. Initial and continued airworthiness.

Modern avionics and ground-based systems implement planning and real-time execution of 4DT functionalities, including multi-objective 4DT optimisation, negotiation and validation in the future $\mathrm{CNS}+\mathrm{A}$ context. The performance of such systems are improved 
This is the author pre-publication version. This paper does not include the changes arising from the revision, formatting and publishing process. The final version that should be used for referencing is:

S. Ramasamy, R. Sabatini and A. Gardi, "Novel Flight Management System for Improved Safety and Sustainability in the CNS+A Context", in proceedings of Integrated Communication, Navigation and Surveillance Conference (ICNS 2015), Herndon, VA, USA, 2015.

by implementing integrity monitoring and augmentation functions fulfilling Required Communication, Navigation and Surveillance Performance (RCP, RNP and RSP), and thus meeting the Required Total System Performance (RTSP) levels in all phases of flight. The automated systems allow the aircraft to fly user-preferred optimal flight paths and thus they limit the intervention of the air traffic controllers to high-level and emergency decision making. The novel airborne and ground ATM systems will provide better and precise airborne navigation services, optimal collision avoidance and aircraft separation assistance, and effective, secure and reliable communication links [5-10].

\section{NG-FMS Architecture}

State-of-the-art Flight Management Systems (FMS) are primarily responsible for providing automated navigation and guidance services from take-off to landing. The hardware components of modern FMS include the Multi Control Display Unit (MCDU) and FM dedicated processor. The software component embedded in the processor performs the following functions:

a. Positioning and navigation algorithms involving a number of multi-sensor data fusion techniques.

b. Guidance computations in terms of lateral and vertical guidance algorithms.

c. Trajectory generation and optimisation algorithms.

d. Short-term and long-term performance computation algorithms.

e. Dual and single FM mode (single and dual) protocols.

f. Processing, sorting and selection of data bases.

g. Built-In-Test Equipment (BITE) and monitoring.

h. Interface management.

The lateral flight planning includes initialisation of Flight Plan (FPLN) and lateral revisions. The same concept applies to the vertical FPLN as well. The main navigation functions include:

a. Selection of navigation modes.

b. Radio navigation functions including manual and auto selection. c. Inertial Reference System initialisation and alignment.

d. Global Navigation Satellite System (GNSS) initialisation. These might include Global Positioning System (GPS), Galileo, GLObalnaya NAvigatsionnaya Sputnikovaya Sistema (GLONASS), BeiDou (or Compass), Indian Regional Navigational Satellite System (IRNSS), Quasi-Zenith Satellite System (QZSS) or a combination of the available constellations.

The traditional databases associated with FMS are magnetic deviation (MAG DEV), performance database (PERBDB) and navigation database (NAGDB). In addition to these databanks, weather, demographic distribution, digital terrain elevation, environmental and pilot modifiable databases are introduced for time based operations and TBO/PBO. The NG-FMS performs comprehensively all the current day FMS functions as listed above and provides autothrottle controls for engines. Additionally, the NG-FMS communicates with a ground-based 4DT Planning, Negotiation and Validation (4-PNV) system, which is part of a Collaborative Decision Making (CDM) network also including Airline Operating Centres (AOC) and Air Navigation Service Providers (ANSP). The NG-FMS is termed as NG-MMS (Next Generation Mission Management System) when tailored for RPAS functions. The NG-FMS/NGMMS software is based on multi-objective and multi-model 4DT optimisation algorithms for strategic, tactical and emergency scenarios. The emergency avoidance of natural and man-made obstacles involves the human pilot and controller with data obtained from multi-senor tracking and data fusion algorithms. Tactical operations (i.e., 4DT intent negotiation and validation with the ATM) are enabled by the Next-Generation Air Data Link (NG-ADL) [8]. Prevention of collisions takes into account weather and airspace sector information in addition to tactical intervention and emergency avoidance tasks.

The key NG-FMS software modules are:

a. Trajectory Planning/Optimisation - This module performs 4DT planning and optimisation functions for pre-tactical, tactical and emergency operations. The 
This is the author pre-publication version. This paper does not include the changes arising from the revision, formatting and publishing process. The final version that should be used for referencing is:

S. Ramasamy, R. Sabatini and A. Gardi, "Novel Flight Management System for Improved Safety and Sustainability in the CNS+A Context", in proceedings of Integrated Communication, Navigation and Surveillance Conference (ICNS 2015), Herndon, VA, USA, 2015.

4DT optimiser includes the models pool and the constraints pool. A number of performance criteria and cost functions are used for optimisation including minimisation of fuel consumption, flight time, operative cost, noise impact, emissions and contrails. The databases include navigation, performance, magnetic deviation and environmental databases. The NG-MMS also includes cooperative and non-cooperative SAA software modules.

b. Trajectory Monitoring - It performs state estimation, calculating the deviations between the active 4DT intents and the estimated/predicted aircraft states.

c. Path Correction - It corrects the path deviation in terms of lateral, vertical and time profiles. The generated steering commands are provided to the guidance module of the NG-FMS.

d. Trajectory Negotiation and Validation - It carries out the process of negotiation that can be initiated by the pilot via the NGFMS, making use of the information available on board, or by 4-PNV system.

e. FMS Performance Manager - It monitors the active 4DT intents for errors and addresses the full set of CNS performance requirements throughout the different phases of 4DT intent negotiation and validation.

f. MMS Integrity Manager - This module is used to generate integrity caution (predictive) and warning (reactive) flags based on inputs from different sensors/systems and predefined decision logics. For instance, the main causes of GNSS signal outage and degradation in flight, namely: antenna obscuration, multipath, fading due to adverse geometry and Doppler shift are identified and modelled to implement integrity thresholds and guidance algorithms in the Avionics-Based Integrity Augmentation (ABIA) system [11-13].

Errors affecting the CNS+A systems (e.g., pseudo-range GNSS observables) are taken into account in evaluating the CNS performances. As illustrated in Figure 1, the integrity analysis module provides inputs to the Integrity Flag Generator (IFG) based on the errors affecting the CNS systems. The IFG uses a set of predefined Caution and Warning Integrity Flags (CIF/WIF) threshold parameters to trigger the generation of both caution and warning flags associated with CNS performance degradations. In case a warning flag is generated, a recapture command is used to trigger the 4DT regeneration and optimisation process.

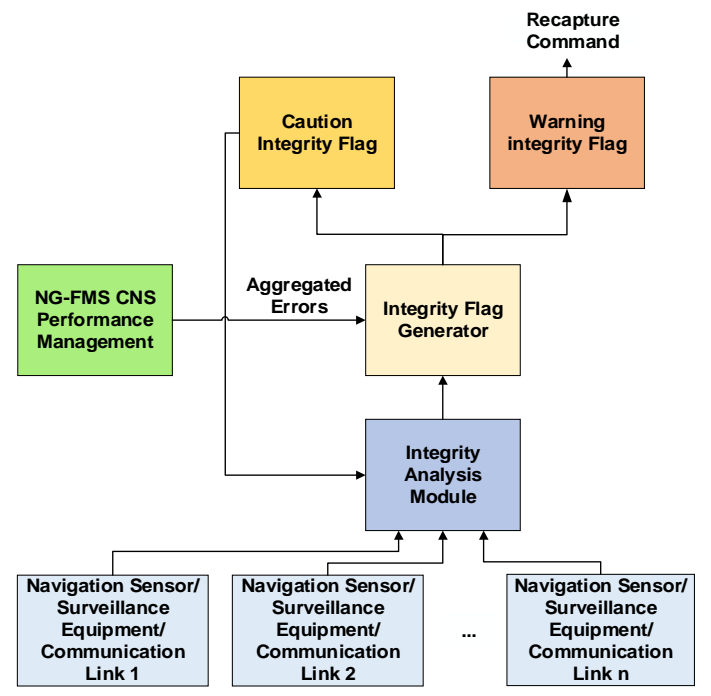

Figure 1. NG-FMS Integrity Monitor

Figure 2 is a schematic block diagram of the NG-FMS performance management modules. The performance management tasks are defined for all CNS+A parameters.

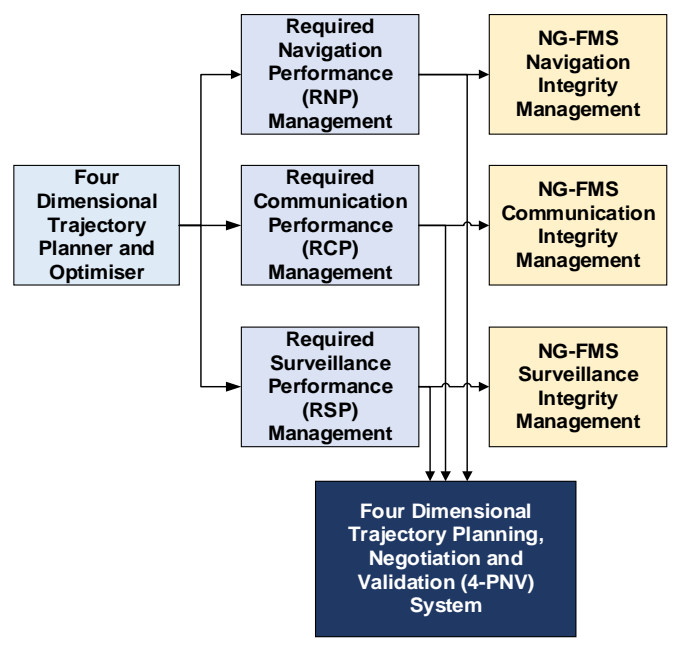

Figure 2. NG-FMS Performance Manager

The Required Navigation Performance (RNP), Required Surveillance Performance (RSP) and Required Communication Performance (RCP) integrity performance management modules provide information to the integrity management software modules that 
This is the author pre-publication version. This paper does not include the changes arising from the revision, formatting and publishing process. The final version that should be used for referencing is:

S. Ramasamy, R. Sabatini and A. Gardi, "Novel Flight Management System for Improved Safety and Sustainability in the CNS+A Context", in proceedings of Integrated Communication, Navigation and Surveillance Conference (ICNS 2015), Herndon, VA, USA, 2015.

are used in-turn to generate usable, timely and valid CIF/WIF. The CNS+A performance management blocks are interfaced with the 4PNV system. Figure 3 is a schematic block diagram illustrating the interaction between NGFMS/NG-MMS and the ground communication network. The overall NG-FMS architecture is illustrated in Figure 4. Airline, airspace, aircraft performance (derived from PERFDB) and ATM operational constraints are taken into account in the trajectory prediction and performance optimisation tasks.

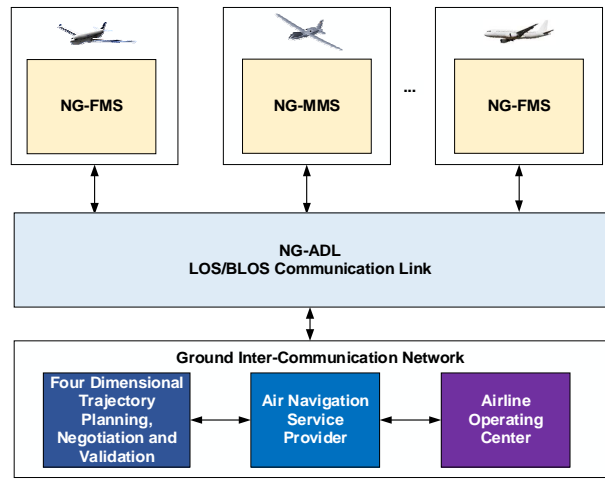

\section{Figure 3. Interaction between NG-FMS and the Ground Systems}

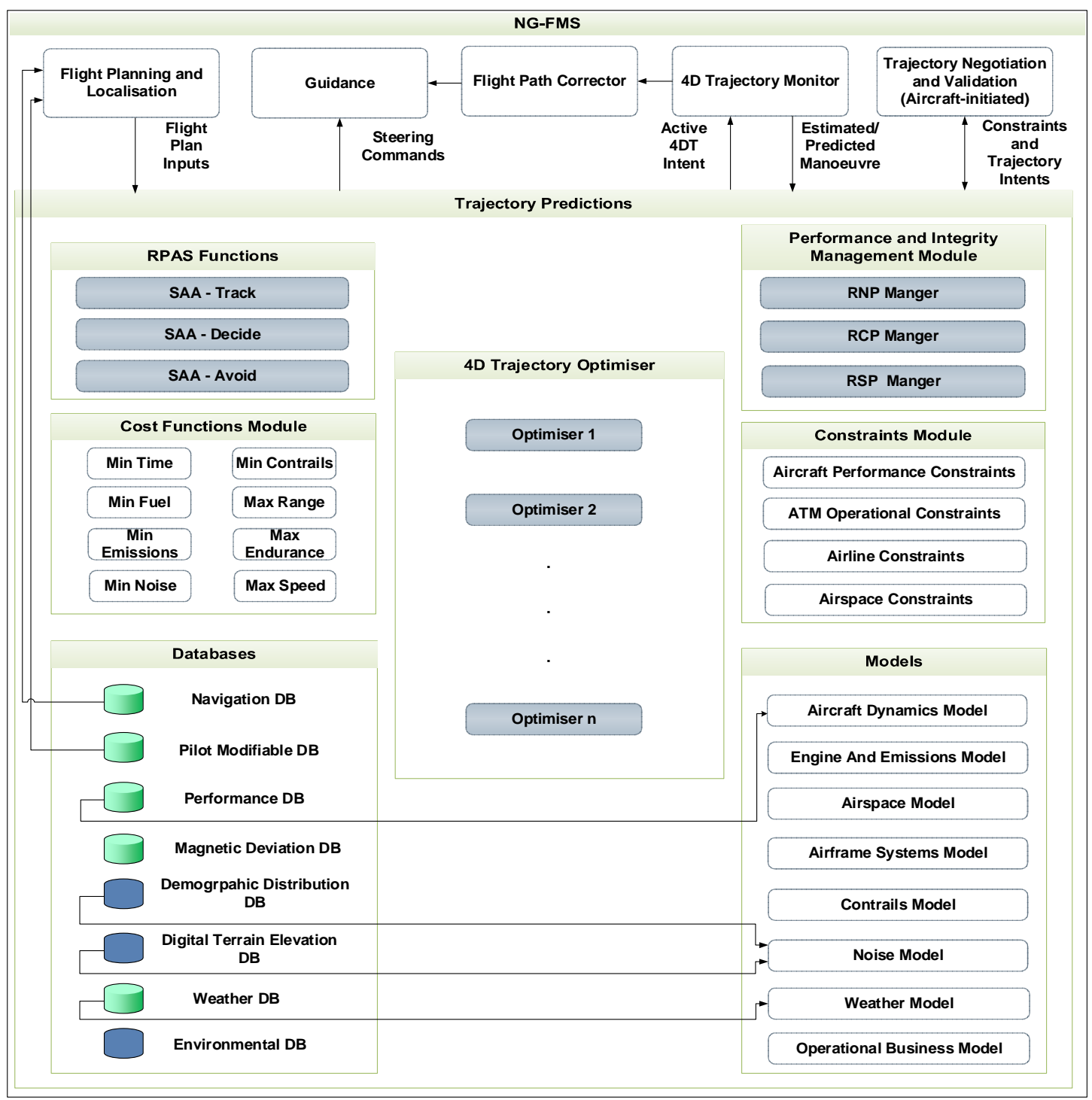

Figure 4. NG-FMS Overall Architecture 
This is the author pre-publication version. This paper does not include the changes arising from the revision, formatting and publishing process. The final version that should be used for referencing is:

S. Ramasamy, R. Sabatini and A. Gardi, "Novel Flight Management System for Improved Safety and Sustainability in the CNS+A Context", in proceedings of Integrated Communication, Navigation and Surveillance Conference (ICNS 2015), Herndon, VA, USA, 2015.

\section{Trajectory Planning}

The NG-FMS generates 4DT intents composed by groups of single trajectories $t$ belonging to the global set T. A number of algorithms have been adopted for trajectory generation and avoidance manoeuvres [14-17]. The consecutive Trajectory Change Points (TCP) along the path are defined with respect to the previous waypoints by conditional probability and generate fly-by and fly-over waypoints for each flight segment up to the destination. The NG-FMS trajectory optimisation algorithms are based on a 3-Degrees-of-Freedom (3$\mathrm{DoF})$ or 6-Degrees-of-Freedom (6-DoF) Aircraft Dynamics Model (ADM) with variable mass. The 3DoF Equations of Motion (EoM) describing the aircraft states and governing the translational movements along the longitudinal, lateral and vertical axes are:

$$
\begin{aligned}
& \frac{\mathrm{d} \varphi}{\mathrm{dt}}=\frac{\mathrm{V} \cos \gamma \sin \chi+\mathrm{V}_{\mathrm{W}_{\varphi}}}{\mathrm{R}_{\mathrm{M}}+\mathrm{h}} \\
& \frac{\mathrm{d} \lambda}{\mathrm{dt}}=\frac{\mathrm{V} \cos \gamma \cos \chi+\mathrm{V}_{\mathrm{W}_{\lambda}}}{\cos \varphi\left(\mathrm{R}_{\mathrm{T}}+\mathrm{h}\right)} \\
& \frac{\mathrm{dh}}{\mathrm{dt}}=V \sin \gamma+\mathrm{V}_{\mathrm{W}_{\mathrm{h}}} \\
& \frac{\mathrm{dV}}{\mathrm{dt}}=\frac{\Delta \mathrm{f}}{\mathrm{m}}-\mathrm{g} \sin \gamma \\
& \frac{\mathrm{d} \gamma}{\mathrm{dt}}=\frac{\mathrm{g}(\mathrm{n} \cos \phi-\cos \gamma)}{\mathrm{V}} \\
& \frac{\mathrm{d} \chi}{\mathrm{dt}}=\left(\frac{\mathrm{N} \cdot \mathrm{g}}{\mathrm{V}}\right)\left(\frac{\sin \phi}{\cos \gamma}\right) \\
& \frac{\mathrm{dm}}{\mathrm{dt}}=-\mathrm{c}(\mathrm{P}, \mathrm{V}, \mathrm{h}) \mathrm{T}(\mathrm{P}, \mathrm{V}, \mathrm{h}) \\
& \Delta \mathrm{f}=\mathrm{T}(\mathrm{P}, \mathrm{V}, \mathrm{h})-\mathrm{D}(\mathrm{L}, \mathrm{V}, \mathrm{h})
\end{aligned}
$$

The above Differential Algebraic Equations (DAEs) incorporate three control variables $\mathrm{u}=(\mathrm{P}, \mathrm{n}, \phi)$ where $\mathrm{P}$ is the engine power setting, $\mathrm{n}$ is the load factor and $\phi$ is the bank angle. The seven state variables are $x=(m, \varphi, \lambda, h, V, \gamma, \chi)$, where $m$ is the aircraft (variable) mass, $\varphi$ is the geodetic latitude, $\lambda$ is the geodetic longitude, $h$ is the altitude, $\mathrm{V}$ is the true air speed, $\gamma$ is the flight path angle, $\chi$ is the heading. $R_{M}$ is the meridional radius of curvature, $\mathrm{R}_{\mathrm{T}}$ is the transverse radius of curvature, $\mathrm{W}$ is the wind velocity, $\mathrm{T}$ is the thrust, $\mathrm{D}$ is the drag and $\mathrm{g}$ is the nominal acceleration due to gravity of the Earth. Assumptions considered are a rigid body aircraft, nil wing bending effect, rigidly mounted aircraft engine on the vehicle body, zero thrust angle, negligible moments of force and inertia, varying mass only as a result of fuel consumption and uniform gravity. Wind effects are considered along the three geodetic reference axes. The geodetic coordinate reference system used is the World Geodetic System of year 1984 (WGS 84). The optimisation problem consists in determining the controls $\mathrm{u}(\mathrm{t})$ and states $\mathrm{x}(\mathrm{t})$ that minimize a performance index, $\mathrm{J}$ given by the sum of Mayer $(\Phi)$ and Lagrange (L) terms as follows:

$$
J=\Phi\left[x\left(t_{0}\right), x\left(t_{f}\right), t\right]+\int_{t_{0}}^{t_{f}} L[x(t), u(t), t] d t
$$

where $t_{0}$ is the initial time and $t_{f}$ is the final time in the time domain. The lateral path is constructed in terms of segments (straight and turns) and is based on the required course change and the aircraft predicted ground speed during the turn. A turn is constructed based on the maximum ground speed during a course change and the turn radius is given by:

$$
\mathrm{T}_{\mathrm{R}}=\frac{\mathrm{GS}}{\mathrm{g} \times \tan (\phi)}
$$

where GS is the maximum ground speed during the turn. The turn arc length is given by:

$$
\mathrm{T}_{\mathrm{AL}}=\text { Course }_{\text {change }} \times \mathrm{T}_{\mathrm{R}}
$$

Given the $T_{R}$, the bank angle is calculated from:

$$
\phi=\arctan \left(\frac{\mathrm{GS}^{2}}{\mathrm{~T}_{\mathrm{R}} \times \mathrm{g}}\right)
$$

The NG-FMS computes turn altitude and speed based on the selected altitude by taking into account the predicted wind at that altitude. The bank angle is determined based on aircraft dynamics and airspace configurations. In order to construct the vertical profile, a number of energy balance equations are typically adopted leading to nominal climb/descent, fixed gradients climb/descent, intermediate speed changes and level flight configurations. The integration steps are constrained by the mission profile imposed altitude, speed and time restrictions as well as performance limitations such as speed and buffet limits, maximum altitude and thrust limits. The data that drives the energy balance equations come from the airframe/engine dependent thrust, fuel flow, drag and air speed schedule models stored in the PERFDB. The vertical profile is obtained from the energy method given by: 
This is the author pre-publication version. This paper does not include the changes arising from the revision, formatting and publishing process. The final version that should be used for referencing is:

S. Ramasamy, R. Sabatini and A. Gardi, "Novel Flight Management System for Improved Safety and Sustainability in the CNS+A Context", in proceedings of Integrated Communication, Navigation and Surveillance Conference (ICNS 2015), Herndon, VA, USA, 2015.

$$
\frac{\mathrm{dh}}{\mathrm{dt}}=\frac{(\mathrm{T}-\mathrm{D}) \mathrm{V}_{\mathrm{T}}}{\mathrm{GW} \times\left(1+\frac{\mathrm{V}_{\mathrm{T}}}{\mathrm{g}} \times \frac{\mathrm{dV_{T }}}{\mathrm{dh}}\right)}
$$

where $\mathrm{V}_{\mathrm{T}}$ is the true air speed and GW is the gross weight. The NG-FMS receives the controlled time of arrival target defined by the 4-PNV system, which becomes the Required Time of Arrival (RTA) to be used by the NG-FMS in determining the optimal trajectory states (final time). The Estimated Time of Arrival (ETA) is assigned to multiple fixes along the flight path. These time metrics gain significance in terms of time based operations. In general, NG-FMS software for all flight phases allow incorporation of a cost index based on a manually entered CAS/Mach or a combined pair. The cost index set by the mission operators is processed by the NG-FMS to set the gain matrix weigh time, fuel, emissions, noise and other costs. The time cost, $\mathrm{J}_{\text {time }}$ is given by:

$$
\mathrm{J}_{\text {time }}=\mathrm{K}_{\mathrm{t}} \mathrm{t}_{\mathrm{f}}
$$

Fuel consumption optimisation is achieved by minimising the difference between the aircraft initial and final mass:

$$
\mathrm{J}_{\text {fuel }}=\mathrm{x}_{7}\left(\mathrm{t}_{\mathrm{f}}\right)-\mathrm{x}_{7}\left(\mathrm{t}_{0}\right)
$$

In terms of aircraft emissions, although engine design and other factors play an influence on the total amount of emission release, engine emission is generally considered as a function of fuel burn, multiplied by a direct emission factor, @. Hence, the mathematical description of the emission rate,

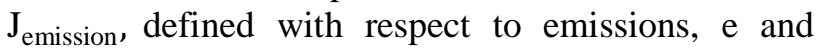
time, $\mathrm{t}$ is given by:

$$
\mathrm{J}_{\text {emission }}=\int_{\mathrm{t}_{0}}^{\mathrm{t}_{\mathrm{f}}} \frac{\mathrm{de}}{\mathrm{dt}}=\left(\mathrm{m}\left(\mathrm{t}_{\mathrm{f}}\right)-\mathrm{m}\left(\mathrm{t}_{0}\right)\right)^{*} \mathrm{Q}
$$

Functions modelling the dependence of $\varrho$ on the engine power setting for turbofan engines were introduced in [17]. The navigation costs, $\mathrm{J}_{\mathrm{C}}$ is given by:

$$
\mathrm{J}_{\mathrm{C}}=\sum_{\mathrm{c}=1}^{\mathrm{N}}\left(\sqrt{\frac{\text { MTOM }_{\text {MTOM }_{\mathrm{avg}}}}{\text { DIS }_{\mathrm{avg}}} \times \mathrm{\text {DIS } _ { \mathrm { c } }}} \times \mathrm{URC}\right.
$$

where MTOM is the Maximum Take-Off Mass, DIS is the horizontal separation between aircraft and intruder, DIS avg depends on the country of implementation and $\mathrm{URCH}$ is the Unitary Rate Charges for air traffic services. These charges were first established by EUROCONTROL for European countries [18]. In order to implement the noise model, demographic distribution and digital terrain elevation data are considered. The terrain proximity cost, $\mathrm{J}_{\mathrm{T}}$ is obtained as a trade-off between an early turn take-off, low path angle climb, low clearance margin from obstacles output and a very steep climb, long straight departure and increased obstacle clearance margin solution [19]. The numerical solution of the trajectory optimisation problem is performed by preliminarily combining the multiple objectives by means of weighted sum, and subsequently solving the combined optimisation problem by means of pseudospectral transcription into a constrained multiphase Non-Linear Programming (NLP) problem.

\section{NG-FMS and AFCS Interactions}

The interactions between NG-MMS and Auto Flight Control System (AFCS) are illustrated in Figure 5. In designing a lateral track control strategy for the RPAS, a viable control strategy is based on the relation [20]:

$$
\frac{\mathrm{X}_{\text {track }}}{\mathrm{k} \times \mathrm{X}_{\text {track }}}=\frac{\mathrm{Y}_{\text {track }}}{\mathrm{Y}_{\text {track }}}
$$

where $X_{\text {track }}$ and $Y_{\text {track }}$ are the current track position of the RPAS with respect to a TCP. The along-track and cross-track velocities are obtained from the airspeed and wind speed velocity vectors. The condition to satisfy the control strategy is given by:

$$
\text { Error }=\left(\mathrm{k} \times \mathrm{X}_{\text {track }} \times \mathrm{Y}_{\text {track }}\right)-\left(\mathrm{Y}_{\text {track }} \times \mathrm{X}_{\text {track }} \cdot{ }^{\cdot}\right)
$$

Proportional-Integral-Derivative (PID) and/or neural network/fuzzy logic based controllers are typically implemented for eliminating the error. In order to study the effects of uncertainties on the generated 4DT, a detailed error analysis is performed. The errors might be due to database accuracy degradations, system modelling errors, atmospheric disturbances and subsystem errors. The system states are modified with the addition of the stochastic term, $\mathrm{e}(\mathrm{t})$ and are represented by:

$$
\dot{x}(t)=f[x(t), u(t), e(t), t]
$$

In order to perform a sensitivity analysis, the sensitivity of a trajectory attribute, $\mathbf{J}$ including consumption of fuel, noise, emissions, etc. is considered with respect to a model parameter, $\Delta$ and is given by:

$$
\Delta=\Delta^{\mathrm{n}}+\mathrm{d} \Delta
$$


This is the author pre-publication version. This paper does not include the changes arising from the revision, formatting and publishing process. The final version that should be used for referencing is:

S. Ramasamy, R. Sabatini and A. Gardi, "Novel Flight Management System for Improved Safety and Sustainability in the CNS+A Context", in proceedings of Integrated Communication, Navigation and Surveillance Conference (ICNS 2015),

Herndon, VA, USA, 2015.

The open loop sensitivity of $\mathrm{J}$ is given by:

$$
\frac{\partial \mathrm{J}}{\partial \Delta}_{\mathrm{OL}}=\frac{\mathrm{J}^{\mathrm{OL}}\left(\Delta^{\mathrm{n}}+\mathrm{d} \Delta\right)-\mathrm{J}^{\mathrm{OL}}\left(\Delta^{\mathrm{n}}\right)}{\mathrm{d} \Delta}
$$

The closed loop sensitivity of $\mathrm{J}$ is given by:

$$
\frac{\partial \mathrm{J}}{\partial \Delta}{ }_{\mathrm{CL}}=\frac{\mathrm{J}^{\mathrm{CL}}\left(\Delta^{\mathrm{n}}+\mathrm{d} \Delta\right)-\mathrm{J}^{\mathrm{CL}}\left(\Delta^{\mathrm{n}}\right)}{\mathrm{d} \Delta}
$$

The values of $\mathrm{J}^{\mathrm{OL}}$ and $\mathrm{J}^{\mathrm{CL}}$ are optimised based on the 4DT optimisation algorithm adopted in the NG-
MMS. Each performance index provides a quantitative measure of the attainment of a specific objective and different objectives are typically conflicting. Thus, the optimisation in terms of two or more objectives typically leads to a number of possible solutions, which are still optimal in a mathematical sense. Therefore, a trade-off analysis based on specific performance weightings is required for the operational implementation of multi-objective 4DT optimisation techniques.

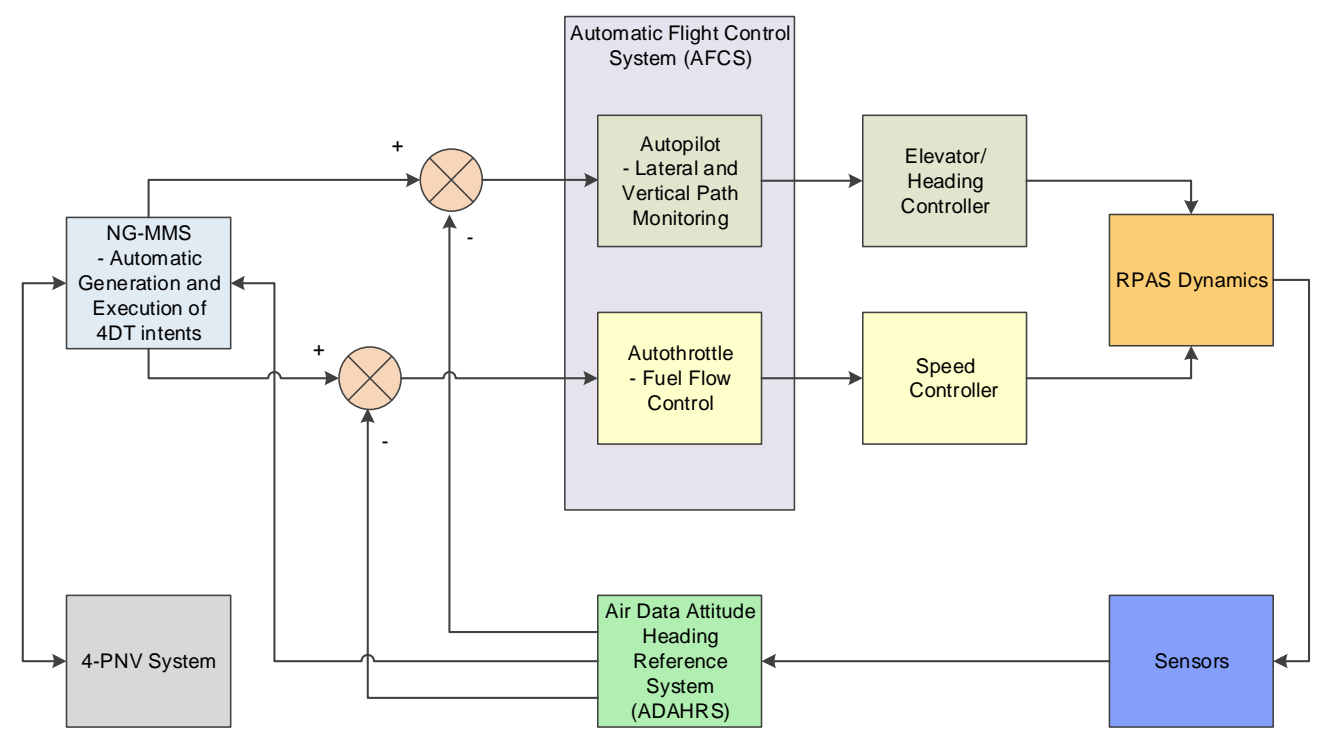

Figure 5. NG-MMS and AFCS Interactions

The errors in aircraft position data are conveniently described by a navigation error ellipsoid. Considering cooperative and noncooperative obstacle avoidance and safe-separation maintenance requirements, the overall uncertainty volume is obtained by adopting a unified approach to SAA [21]. In this approach the navigation error ellipsoid of the host manned aircraft/RPAS and the tracking error ellipsoid of the intruder/obstacle are combined to obtain the overall uncertainty volume. The solution is obtained by translating the errors to unified range and bearing uncertainty descriptors, which apply to both cooperative and non-cooperative scenarios. Modelling the navigation, communication and surveillance errors as white Gaussian noise, the associated probability density functions are:

$$
f_{R T S P}(x)=\frac{1}{\sqrt{2 \pi} \sigma_{C N S}} e^{-\frac{x^{2}}{2 \sigma_{C N S}^{2}}}
$$

where $\sigma_{C N S}$ represents the standard deviation values resulting from errors in communication, navigation and surveillance data $\left(\sigma_{\text {Comm }}, \sigma_{\text {Nav }}, \sigma_{\text {Sur }}\right)$ respectively. The environmental performance of the NG-FMS requires additional data to be included in the existing databases or including a separate database exclusively for evaluating environmental sustainability functions. Noise abatement operational procedures are in use today and include noise abatement flight procedures including Continuous Descent Arrival (CDA), Noise Abatement Departure Procedures (NADP), modified approach angles, staggered, or displaced landing thresholds, low power/low drag approach profiles and minimum use of reverse thrust after landing, etc. The RTA 
This is the author pre-publication version. This paper does not include the changes arising from the revision, formatting and publishing process. The final version that should be used for referencing is:

S. Ramasamy, R. Sabatini and A. Gardi, "Novel Flight Management System for Improved Safety and Sustainability in the CNS+A Context", in proceedings of Integrated Communication, Navigation and Surveillance Conference (ICNS 2015),

Herndon, VA, USA, 2015.

algorithms involve the variation in speed as the lateral profile and cruise altitude are fixed idle-thrust performance path. According to the updated SESAR Implementation Master Plan [22], the foreseen future deployments evolve from radar-based operations to $\mathrm{TBO} / \mathrm{IBO}$ to achieve performance-driven ATM in all airspace domains. In this context, the NG-FMS acts as a key enabler to implement the operational concept and integrate the pre-emptive $\mathrm{CNS}+\mathrm{A}$ technologies. Green trajectories, based on more precise, reliable and predictable three dimensional flight path, optimized for minimum noise impact and low emission, include agile trajectory management, in response to any meteorological hazard. A green mission from start to destination, with management of new climb, cruise and descent profiles, allows multicriteria optimization (noise, emissions, fuel, time), including management of weather conditions which could negatively impact the aircraft optimal route and result in additional fuel consumption.

\section{Simulation Case Studies}

Simulation case studies are carried out to illustrate the capability of NG-FMS to generate and optimise 4DT intents. 4DT intents are generated by the NG-FMS and their optimisation is accomplished with respect to time and fuel costs. Noise Sensitive Areas (NSA) are considered in the simulation as path constraints in the climb phase and weather cells are taken into account in the cruise phase. A non-rotating spherical Earth model is employed in the simulation. A typically loaded Airbus A380 aircraft with maximum take-off weight of $420,000 \mathrm{~kg}$ is assumed to be flying the mission. The aircraft parameters and aerodynamic data are extracted from the EUROCONTROL Base of Aircraft Data (BADA) [23]. The simulations are executed on a Windows 7 Professional platform (64-bit OS) supported by an Intel Core i7-4510 CPU with clock speed $2.6 \mathrm{GHz}$ and $8.0 \mathrm{~GB}$ RAM. The aircraft takes off from London Heathrow airport (International Civil Aviation Organization - ICAO code: EGLL) and proceeds towards the AMRAL waypoint in the United Kingdom while climbing up to the planned cruise flight level 330 (33,000 feet). Two NSA are introduced as path constraints in this first simulation case study. Figure 6 is a 3D view of the several different trajectories generated, each resulting from a different cost function for time and fuel minimisation.
In order to account for environmental objectives, the numerical data for time, fuel burn and associated emissions are analysed. The time, fuel burn and emissions $\left(\mathrm{CO}_{2}\right.$ and $\left.\mathrm{NO}_{\mathrm{x}}\right)$ change, as a result of trajectory variations indicate that for every kilogram of kerosene fuel burn, $3.13 \mathrm{~kg}$ of $\mathrm{CO}_{2}$ and $0.014 \mathrm{~kg}$ of NOx (as the average of NOx emission below and above FL 330) are generated [9].

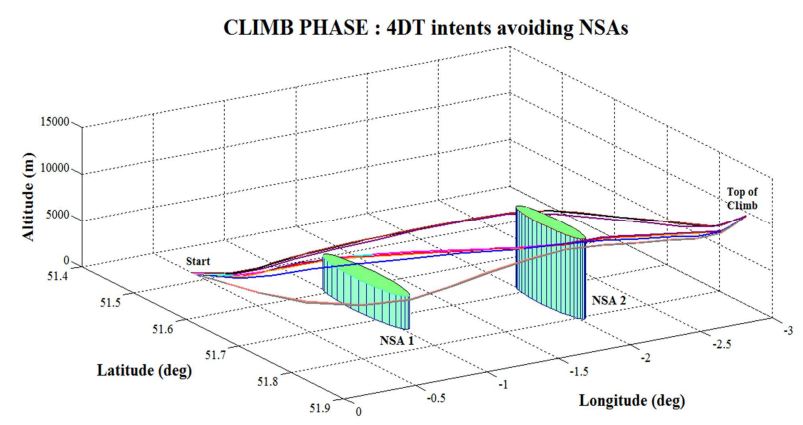

Figure 6. Climb Phase 4DT Intents

In the cruise phase, two weather cells are inserted and the 4DT intents are able to avoid those areas as illustrated in Figure 7. The results demonstrate that the insertion of NSAs and weather cells directly into the cost function is feasible both numerically and analytically. The trajectory intents optimised with contrails and weather cells as path constraints are generated within 10 seconds in the hardware platform mentioned earlier.

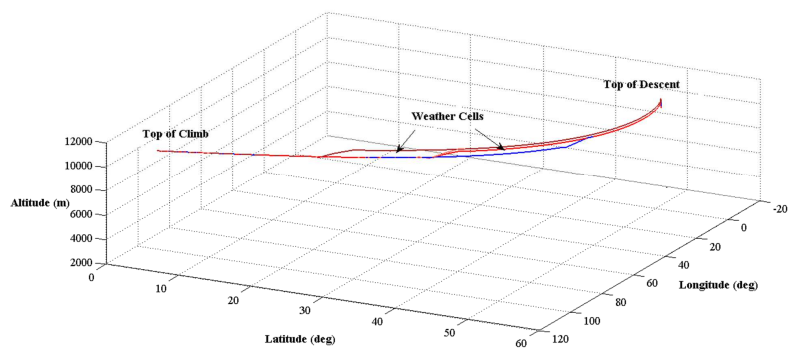

Figure 7. Cruise Phase 4DT Intents

The trajectory with minimum fuel burn consumes $700 \mathrm{~kg}$ fuel less than that of the trajectory for minimum time. The optimisation of the reference trajectory took 15 seconds while the NSA avoidance trajectory required an additional 10 seconds for optimisation. During the descent and arrival phases, the aircraft proceeds from the top of descent waypoint to Kuala Lumpur, Malaysia (ICAO code: WMKK). In the absence of suitable NG-FMS autopilot loops, the trajectory affected by wind might exceed the RNP 
This is the author pre-publication version. This paper does not include the changes arising from the revision, formatting and publishing process. The final version that should be used for referencing is:

S. Ramasamy, R. Sabatini and A. Gardi, "Novel Flight Management System for Improved Safety and Sustainability in the CNS+A Context", in proceedings of Integrated Communication, Navigation and Surveillance Conference (ICNS 2015), Herndon, VA, USA, 2015

0.5 containment boundaries. However, the NG-FMS raises a CIF after detecting the potential RNP violation due to wind in the descent path. This CIF triggers a new intent optimisation/negotiation loop with the 4-PNV system and thus prevents the production of a WIF. In the conditions described, the time required to generate the CIF is 5 seconds [9]. To avoid a WIF event, an NG-FMS-autopilot loop is performed based on the interactions with AFCS allowing re-insertion into the original nominal trajectory immediately after a CIF event.

\section{Human Machine Interface and Interaction $\left(\mathbf{H M I}^{\mathbf{2}}\right)$}

Electronic Flight Instrument System (EFIS) displays are the Primary Flight Display (PFD) and Navigation and Tactical Display (NTD). EFIS displays enable continuous monitoring of flight information and parameters and allow the pilots to implement corrective measures if required. The PFD provides information on thrust and control commands generated by the FMC. Track of FPLN, instantaneous position, Distance-To-Destination (DTD) based waypoints based on flight phases, segments and sections are displayed on the NTD. Other relevant information including navigation aids, airports, altitude/speed/time constraints and wind information are also displayed in the NTD. In order to realise time based operations, the key parameters for 4DT operations have to be identified and included in trajectory predictions component of the current FMS systems. Furthermore, functions and formats that support full 4D information, and human factors consideration for evaluation of the 4DT information have to be analysed considering mixed equipage. Novel forms of $\mathrm{HMI}^{2}$ are required for the NG-FMS in order to enable time based operations. Furthermore, recent research activities have been performed to identify the specific requirements for single-pilot and RPAS operations as well. The $\mathrm{HMI}^{2}$ aspects in the case of NG-MMS in a Ground Control Station (GCS) will vary significantly from the manned version, wherein the control shifts from a pilot to system operator perspective. As pointed out in STAGNAG 4586, there is a need of greater levels of interoperability and therefore a significant evolution of the $\mathrm{HMI}^{2}$ design is required [24]. The new formats and functions development activities are focussing on: a. Navigation, guidance and autopilot functions.

b. Radio communication functions.

c. Vehicle configuration data including sensors information (especially in the case of RPAS).

d. Monitoring of integrity flags.

e. Monitoring of CNS+A performance evaluation metrics.

\section{Conclusions and Future Work}

The system architecture of a novel NG-FMS was presented, providing 4-Dimensional Trajectory Optimisation (4DT-O) and air-to-ground trajectory negotiation/validation functionalities in synergy with NG-ATM systems to support Trajectory/Intent based operations (TBO/IBO). Suitable mathematical models were described for trajectory planning tasks. Simulation case studies were performed and the results demonstrated the functional capability of the flight/mission management system to generate costeffective 4D trajectory intents satisfying operational and environmental constraints. Future research will address the uncertainty analysis in detail and will incorporate suitable navigation and tracking algorithms for cooperative and non-cooperative SAA. Additionally, CNS ABIA algorithms will be incorporated as an integral part of both flight and mission management systems for a variety of applications. Data driven architectures and networked System of Systems (SoS) concepts for implementing the NG-FMS (and NG-MMS) are also being explored. Additionally, data link requirements in high density air traffic scenarios are being considered, including the associated trajectory data descriptors and negotiation/validation protocols.

\section{References}

[1] Logan MJ, Chu J, Motter MA, Carter DL, Ol M, Zeune C, Small UAV Research and Evolution in Long Endurance Electric Powered Vehicles, AIAA Infotech@ Aerospace 2007 Conference and Exhibit, AIAA Paper, pp. 7-10, 2007.

[2] Martin TL, Campbell DA, RPAS integration within an Australian ATM system: What equipment and which airspace, 2014 International Conference on Unmanned Aircraft Systems (ICUAS), pp. 656-668, 2014.

[3] Amato G, EUROCAE WG-73 on Unmanned Aircraft Systems, ed: EUROCAE. 
This is the author pre-publication version. This paper does not include the changes arising from the revision, formatting and publishing process. The final version that should be used for referencing is:

S. Ramasamy, R. Sabatini and A. Gardi, "Novel Flight Management System for Improved Safety and Sustainability in the CNS+A Context", in proceedings of Integrated Communication, Navigation and Surveillance Conference (ICNS 2015), Herndon, VA, USA, 2015.

[4] JAA, UAV TASK-FORCE Final Report - A concept for European regulations for civil unmanned aerial vehicles (UAVs), 2004.

[5] Ramasamy S, Sabatini R, Gardi A, Kistan T, Next Generation Flight Management System for Real-Time Trajectory Based Operations, Applied Mechanics and Materials, vol. 629, Trans Tech Publications. Switzerland, pp. 344-349, 2014. doi:10.4028/www.scientific.net/AMM.629.344

[6] Ramasamy S, Sangam M, Sabatini R, Gardi A, Flight Management System for Unmanned Reusable Space Vehicle Atmospheric and Re-entry Trajectory Optimisation, Applied Mechanics and Materials, vol. 629, Trans Tech Publications, Switzerland, pp. 304309. 2014. doi: 10.4028/www.scientific.net/ AMM.629.304

[7] Sullivan D, Totah J, Wegener S, Enomoto F, Frost C, Kaneshige J, Frank J, Intelligent Mission Management for Uninhabited Aerial Vehicles, NASA Ames Research Center, Moffett Field, CA, USA.

[8] Gardi A, Sabatini R, Ramasamy S, de Ridder K, 4-Dimensional Trajectory Negotiation and Validation System for the Next Generation Air Traffic Management, Proceedings of the AIAA Guidance, Navigation \& Control Conference, Boston, Massachusetts USA, August 2013. doi: 10.2514/6.2013-4893.

[9] Ramasamy S, Sabatini R, Gardi A, Liu Y, Novel Flight Management System for Real-Time 4Dimensional Trajectory Based Operations, Proceedings of the AIAA Guidance, Navigation \& Control Conference, Boston, Massachusetts, USA, August 2013. DOI: 10.2514/6.2013-4763

[10] Camilleri W, Chircop K, Zammit-Mangion D, Sabatini R, Sethi V, Design and Validation of a Detailed Aircraft Performance Model for Trajectory Optimization, AIAA Modeling and Simulation Technologies Conference 2012 (MST 2012), Minneapolis, Minnesota (USA), August 2012. DOI: 10.2514/6.2012-4566

[11] Sabatini R, Moore T, Hill C, A New AvionicsBased GNSS Integrity Augmentation System: Part 1 - Fundamentals, The Journal of Navigation, vol. 66, no. 3, pp. 363-384, 2013. doi: 10.1017/S037346331 3000027
[12] Sabatini R, Moore T, Hill C, A New Avionics Based GNSS Integrity Augmentation System: Part 2 - Integrity Flags, The Journal of Navigation, vol. 66, No. 4, pp. 511-522, June 2013. doi: 10.1017/S0373463313000143

[13] Sabatini R, Moore T, Hill C, Assessing GNSS Integrity Augmentation Techniques in UAV Senseand-Avoid Architectures, Proceedings of the $16^{\text {th }}$ Australian International Aerospace Congress (AIAC16), Melbourne, Australia, 2015.

[14] Sammut M, Zammit-Mangion D, Sabatini R, Optimization of Fuel Consumption in Climb Trajectories using Genetic Algorithm Techniques, AIAA Guidance, Navigation \& Control Conference 2012 (GNC 2012), Minneapolis, Minnesota (USA), August 2012. DOI: $10.2514 / 6.2012-4829$

[15] Gu W, Navaratne R, Quaglia D, Yu Y, Chircop K, Madani I, Jia H, Sethi V, Sabatini R, ZammitMangion D, Towards the Development of a Multidisciplinary Flight Trajectory Optimization Tool GATAC, ASME Turbo Expo 2012 Conference, Copenhagen (Denmark), June 2012. DOI: 10.1115/GT2012-69862

[16] Gardi A, Sabatini R, Ramasamy S, and Kistan T, "Real-Time Trajectory Optimisation Models for Next Generation Air Traffic Management Systems", Applied Mechanics and Materials, vol. 629, pp. 327332, 2014. DOI: 10.4028/www.scientific.net/ AMM.629.327

[17] Gardi A., Sabatini R., Ramasamy S., and Marino M., "Automated ATM system for 4dimensional trajectory based operations", in proceedings of 16th Australian International Aerospace Congress (AIAC16), Melbourne, Australia, 2015. DOI: 10.13140/2.1.3635.0244

[18] Unit Rates and Tariffs, Central Route Charge Office, EUROCONTROL [Available Online].

[19] Peter O.T, Constantinescu C.E, Stefanescu I.B, Objective Function for Trajectory Optimisation in Trajectory Based Operations, AIAA Guidance, Navigation and Control Conference, Chicago, Illinois, USA, 2009.

[20] Marius N, Lateral track control law for AEROSONDE UAV, $39^{\text {th }}$ Aerospace Sciences Meeting and Exhibit, American Institute of Aeronautics and Astronautics, 2001. 
This is the author pre-publication version. This paper does not include the changes arising from the revision, formatting and publishing process. The final version that should be used for referencing is:

S. Ramasamy, R. Sabatini and A. Gardi, "Novel Flight Management System for Improved Safety and Sustainability in the CNS+A Context", in proceedings of Integrated Communication, Navigation and Surveillance Conference (ICNS 2015), Herndon, VA, USA, 2015.

[21] Ramasamy S, Sabatini R, Gardi A, Towards a Unified Approach to Cooperative and NonCooperative RPAS Detect-and-Avoid, Fourth Australasian Unmanned Systems Conference 2014 (ACUS 2014), Melbourne, Australia. DOI: 10.13140/2.1.4841.3764

[22] SESAR JU, European ATM Master Plan, Edition 2, Brussels, Belgium, 2012.
[23] EUROCONTROL, User Manual for the Base of Aircraft Data (BADA), Revision 3.8, 2000.

[24] STAGNAG 4586, Standard Interfaces of UAV Control System (UCS) for NATO UAV Interoperability, Edition 2, 2007.

2015 Integrated Communications Navigation and Surveillance (ICNS) Conference

April 21-23, 2015 